\title{
Evaluation of Various Factors Affecting Emergency Intubation
}

\author{
Meenakshi K. Moorthy ${ }^{1}$ \\ ${ }^{1}$ Assistant Professor, Department of Anaesthesia, Hind institute of Medical Sciences, Ataria, Sitapur, Uttar Pradesh, India
}

Abstract

Background: Inadequate airway management is a major contributor to pre-hospital morbidity and mortality. The present study was conducted to evaluate various factors affecting endotracheal intubation. Subjects and Methods: This study was conducted on 474 patients requiring ETI. Informed consent was obtained from family members of all patients. Emergency intubation was performed as a 'rapid sequence induction' (RSI), with preoxygenation and cricoid pressure followed by an induction agent and then suxamethonium. Factors affecting ETI was recorded. Results: Out of 476 patients, males were 280 and females were 196. Indication of RTI was low GCS seen in 64, injury in 320, respiratory insufficiency in 10 and cardiac arrest in 82. The difference was significant $(\mathrm{P}<0.05)$. Method of ETI was RSI in 290, without relaxant in 81 and without any medication in 105. The difference was significant (P-0.05). Common difficulties in ETI were blood seen in 30\%, vomit in $14 \%$, hypersalivation in $28 \%$, anatomical difficulties in $7 \%$, patient position in $10 \%$ and technical problems in $11 \%$. The difference was significant $(\mathrm{P}<0.05)$. Conclusion: The presence of blood, vomit, hypersalivation, anatomical difficulties, patient position and technical problems are among various factors affecting ETI.

Keywords: Airway management, Endotracheal intubation, technical.

Corresponding Author: Dr. Meenakshi K. Moorthy, Assistant Professor, Department of Anaesthesia, Hind institute of Medical Sciences, Ataria, Sitapur, Uttar Pradesh, India.

Received: May 2019

Accepted: May 2019

\section{Introduction}

Inadequate airway management is a major contributor to prehospital morbidity and mortality. Several studies examining pre-hospital deaths from trauma in the UK have shown that airway obstruction was thought to have contributed to death from major trauma in up to $85 \%$ of patients. On the other hand, several studies have shown that trauma patients may profit from a definitive airway control by pre-hospital endotracheal intubation (ETI). ${ }^{[1]}$

Endotracheal intubation (ETI) is a rapid, simple, safe and non surgical technique that achieves all the goals of airway management such as maintains airway patency, protects the lungs from aspiration and permits leak free ventilation during mechanical ventilation, and remains the gold standard procedure for airway management.1When ETI is difficult or has failed both elective airway management as well as emergency airway management are alternatives to ETI. ${ }^{[2]}$

The knowledge, technical skills and crisis management capabilities of the anesthesiologists play a vital role in the occurrence and outcome of complications during airway management. A hurried intubation, without adequate evaluation of the airway or preparation of the patient or the equipment is more likely to cause damage. ${ }^{[3]}$ Devices such as laryngeal mask airway and the combitube may be used. Both ETI and the use of the other airways are associated with complications, some of them life threatening. It is essential for anesthesiologists to be aware of these complications, and to have an effective strategy to prevent and manage these complications when they arise. ${ }^{[4]}$ The present study was conducted to evaluate various factors affecting endotracheal intubation.

\section{Subjects and Methods}

This study was conducted in the department of Anesthesiology. It comprised of 474 patients requiring ETI. Informed consent was obtained from family members of all patients.

Data pertaining to patient was recorded in case history performa. Emergency intubation was performed as a 'rapid sequence induction' (RSI), with preoxygenation and cricoid pressure followed by an induction agent and then suxamethonium. Patients with cardiac arrest and patients with glasgow-Coma-Scale 3 were intubated without any medication. Factors affecting ETI was recorded. Results were tabulated and subjected to statistical analysis. P value < 0.05 was considered significant.

\section{Results}

Table 1: Distribution of patients

Table 1: Distribution of patients
\begin{tabular}{|c|c|c|}
\hline \multicolumn{3}{|c|}{ Total- 476 } \\
\hline Gender & Males & Females \\
\hline No. & 280 & \\
\hline
\end{tabular}


[Table 1] shows that out of 476 patients, males were 280 and females were 196.

Table 2: Indication and method of ETI

\begin{tabular}{|l|l|l|}
\hline Indication of ETI & Number & \multirow{2}{*}{0.01} \\
\hline Low GCS & 64 & \\
\hline Pattern of injury & 320 & \\
\hline Respiratory insufficiency & 10 & \\
\hline Cardiac arrest & 82 & \\
\hline Method of ETI & & \multirow{2}{*}{0.02} \\
\cline { 1 - 2 } RSI & 290 & \\
\cline { 1 - 2 } Without relaxant & 81 & \\
\cline { 1 - 2 } Without any medications & 105 & \\
\hline
\end{tabular}

[Table 2] shows that indication of RTI was low GCS seen in 64 , injury in 320 , respiratory insufficiency in 10 and cardiac arrest in 82. The difference was significant $(\mathrm{P}<0.05)$. Method of ETI was RSI in 290, without relaxant in 81 and without any medication in 105 . The difference was significant (P-0.05).

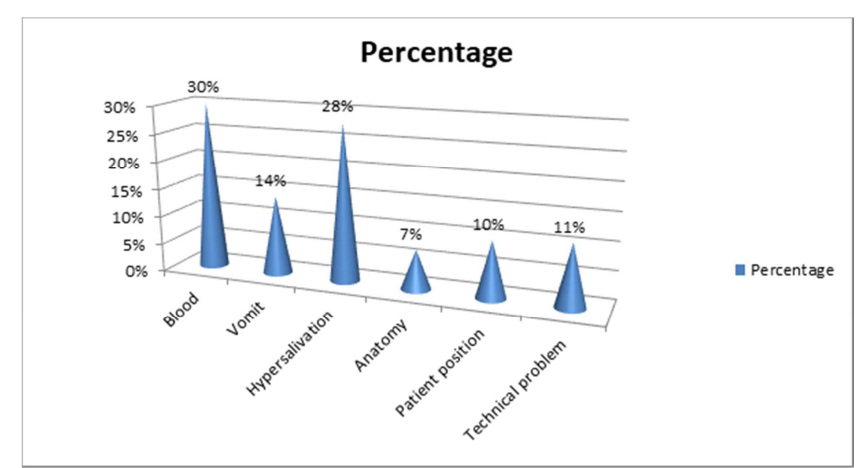

Figure 1: Difficulty in ETI in patients

[Figure 1] shows that common difficulties in ETI were blood seen in $30 \%$, vomit in $14 \%$, hypersalivation in $28 \%$, anatomical difficulties in $7 \%$, patient position in $10 \%$ and technical problems in $11 \%$. The difference was significant $(\mathrm{P}<0.05)$.

\section{Discussion}

Pre-hospital airway management is more difficult because of a number of factors, such as limited equipment and monitoring, lack of skilled help, blood, vomit and debris in the upper airway, inadequate lighting, excessive noise and impaired patient access. ${ }^{[5]}$ Therefore, pre-hospital airway management is difficult, even for anesthetists with extensive experience in airway management.

The present study was conducted to evaluate various factors affecting endotracheal intubation.

In present study, out of 476 patients, males were 280 and females were 196. This is in agreement with Karch et al. ${ }^{[6]}$ In our study indication of RTI was low GCS seen in 64, injury in 320, respiratory insufficiency in 10 and cardiac arrest in 82 . The difference was significant $(\mathrm{P}<0.05)$. Method of ETI was RSI in 290, without relaxant in 81 and without any medication in 105 . Wang et al, ${ }^{[7]}$ conducted a study in which 342 patients $(9.3 \%)$ underwent ETI. The overall success rate was $100 \%$; in $87.4 \%$ the first attempt was successful, whereas in $11.1 \%$ a second and in $1.5 \%$ a third ETI attempt was necessary. No patient required a surgical intervention. Limited access to the patient was found upon arrival at the scene in $20.2 \%$ of the patients and in $9.6 \%$ of the patients at the time of ETI attempt. An orotracheal ETI technique was used in all patients. In the patients in whom only one ETI attempt was necessary for successful intubation, the assessment of ETI conditions was rated 'very good' or 'good' in $94.7 \%$, but in those requiring a second or third ETI attempt this was reduced to 68.6 and $20.0 \%$, respectively. Difficulties encountered during ETI included blood (19.9\%), vomit/debris (15.8\%) and secretions (13.8\%) in the upper airway; anatomical reasons $(11.7 \%)$, patient position $(9.6 \%)$ and surrounding conditions $(9.1 \%)$, making laryngoscopy more difficult.

We found that common difficulties in ETI were blood seen in $30 \%$, vomit in $14 \%$, hypersalivation in $28 \%$, anatomical difficulties in $7 \%$, patient position in $10 \%$ and technical problems in $11 \%$. Orleugart et al, ${ }^{[8]}$ found bright ambiet light and trauma related anatomical changes as one of the leading difficulties in ETI.

The difficult airway and failed intubation encompass a spectrum including difficult mask ventilation, difficult laryngoscopy, difficult intubation and failed intubation. The most dreaded situation is cannot-ventilate-cannot- intubate (CVCI) situation in an apnoeic anaesthetized patient. This is a life threatening emergency occurring in about 1 in 10,000 anaesthetics. Failure to achieve oxygenation will result in death or hypoxic brain damage. ${ }^{[14]}$ Repeated attempts at intubation result in more morbidity, and the number of attempts should be restricted to $3 .{ }^{[9]}$

The main reasons mentioned by paramedics for unsuccessful field intubation in previous US studies have been patient gagging and patient combativeness or trismus in up to $38 \%$ of the cases. The choice of sedation protocol for airway control in critically ill patients remains a source of controversy. The limitation of the present study is small sample size. ${ }^{[10]}$

\section{Conclusion}

Authors found that presence of blood, vomit, hypersalivation, anatomical difficulties, patient position and technical problems are among various factors affecting ETI.

\section{References}

1. Winchell RJ, Hoyt DB. Endotracheal in the field improves survival in patients with severe head injury. Arch Surg 1997; 132: 592-7.

2. Deakin CD. Anaesthetists are best people to provide pre-hospital airway management. Br Med J. 2000; 320: 1006-9.

3. Tortella BJ. Airway management. Emerg Care Quarterly. 1991; 7: 112.

4. Pepe PE, Compass MK, Joyce TH. Pre-hospital endotracheal intubation: rational for training emergency personell. Ann Emerg Med. 1985; 14: 1085-92.

5. Hussain LM, Redmond AD. Are pre-hospital deaths from accidental injury preventable? Br Med J. 1994; 308: 1077-80.

6. Karch SB, Lewis T, Young S. Field intubation of trauma patients: complications, indications and outcomes. Am J Emerg Med 2001; 37: 32-7.

7. Wang HE, O'Connor RE, Megargel RE. The utilization of midazolam as a pharmacologic adjunct to endotracheal intubation by paramedics. Prehosp Emerg Care. 2000; 4: 14-18

8. Adnet F, Jouriles NJ, Le Toumelin P. Survey of out-of-hospital 


\section{Maarthy : Emergency intulbation}

0

emergency intubations in the French pre-hospital medical system: a multicenter study. Ann Emerg Med 1998; 32: 454-60

9. Orliaguet G, Tartiere S, Lejay M. Prospective in-field evaluation of orotracheal intubation by emergency medical services physicians. J
Europeen des urgencies 1997; 1: 27-32.

10. Mackay CA, Terris J, Coats TJ. Pre-hospital rapid sequence induction by emergency physicians: is it safe? Emerg Med J. 2001; 18: 20-4.

Copyright: (c) the author(s), publisher. Academia Anesthesiologica Internationalis an Official Publication of "Society for Health Care \& Research Development". It is an open-access article distributed under the terms of the Creative Commons Attribution Non-Commercial License, which permits unrestricted non-commercial use, distribution, and reproduction in any medium, provided the original work is properly cited.

How to cite this article: Moorthy MK. Evaluation of Various Factors Affecting Emergency Intubation. Acad. Anesthesiol. Int. 2019;4(2):1-3. DOI: dx.doi.org/10.21276/aan.2019.4.2.1

Source of Support: Nil, Conflict of Interest: None declared. 